INVESTIGACIÓN/RESEARCH

\title{
VALLADOLID COMO DESTINO ENOTURÍSTICO
}

Mónica Matellanes Lazo1: Universidad Europea Miguel de Cervantes de Valladolid. España.

$\underline{\text { mmatellanes@uemc.es }}$

\section{RESUMEN}

Numerosas ciudades han visto ya las ventajas que reportan las actividades enológicas. Valladolid se intenta posicionar como una metrópoli ideal para el destino enoturístico, cultural y para la realización de eventos. La investigación que se muestra pretende dar a conocer el mundo del enoturismo y acercarlo a un mayor número de personas de forma llamativa e interactiva. Se trata de valorar la ciudad de Valladolid como destino enoturístico a nivel mundial, nacional, regional y provincial. Para ello se ha contactado con profesionales del sector para que ofrezcan sus opiniones y argumentaciones justificadas sobre Valladolid como ciudad festival del Vino. Los resultados afirman que por los aspectos valorados, sí es adecuada esta ciudad para albergar un importante evento sobre la temática estudiada.

PALABRAS CLAVE: Valladolid - Vino - Enoturismo - Marketing - Comunicación Evento

\footnotetext{
${ }^{1}$ Autor correspondiente:

Mónica Matellanes Lazo: Profesora Doctora Agregada de la Universidad Europea Miguel de Cervantes de Valladolid. España. Experta en Marketing y Comunicación del Enoturismo.

Correo: mmatellanes@uemc.es
} 


\title{
VALLADOLID AS ENOLOGICAL-TOURISM DESTINY
}

\begin{abstract}
Some cities in the world have seen the advantages of Enological-enoturism's activities. Valladolid city try to achieve a market positioning as an ideal metropolis to be an enological, culture destiny and city of wine event festival. The research pretends to show the wine sector and enological-tourism to many people with a spectacular and interactive way. It pretends to value Valladolid as a city able to develop enologicaltourism's activities in the world. For that we contacted with professionals of the area to obtain arguments and answers about Valladolid as a city of wine festival. The results explain that Valladolid is good for doing these activities and to be considered a city of wine.
\end{abstract}

KEY WORDS: Valladolid - Wine - Enological-tourism - Marketing - Communication - Event

\section{INTRODUCCIÓN}

El vino ha sido siempre un protagonista de nuestra civilización, siendo mucho más que un elemento nutritivo y un recurso económico. Hay quien llega a afirmar, como Philippe Faure- Brac, ${ }^{2}$ que el vino es una verdadera religión: sin plantear ahora la relación intrínseca del vino con misterios y ritos, recordemos que el término latino religare significa unir, enlazar, vincular; y ésta ha sido siempre la función del vino en toda la extraordinaria riqueza de sus manifestaciones.

Por un parte, para Valladolid la realización de un evento de estas magnitudes la beneficiaría en todos los aspectos, ya que, afectaría a toda su área de influencia y a la comunidad de Castilla y León. La dimensión de este evento haría posible que Valladolid se considerase como una marca-destino para destacar, no solo a Castilla y León, sino muy especialmente a Valladolid en el contexto que nos encontramos de constante saturación de ofertas.

Por otro lado, se considera que la elección de Valladolid como lugar para albergar este evento, proporcionaría numerosos puestos de trabajo, ayudando además a reducir la despoblación existente en Castilla y León que es uno de los problemas más importantes y acuciantes que tiene nuestra comunidad autónoma.

El impacto que supondría la organización de un evento de estas características en Valladolid habría que considerarlo, entre otros, desde tres puntos de vista: consecuencias demográficas, económicas y culturales que se dejarían sentir inmediatamente en la ciudad:

2 Philippe Faure- Brac: es un sumiller francés. Nacido en 1960. Ganó el premio al mejor sumiller del mundo en 1992. 
Demográficas: el traslado de población de Castilla y León y resto de España hacia Valladolid. Estos movimientos son causados generalmente por motivos socioeconómicos, donde grupos más o menos masivos de personas se instalan de manera provisional, estacional o en definitiva para encontrar una mejor calidad de vida.

Económicas: la actividad económica se desplazaría a Valladolid ya que supone un reclamo la realización de grandes eventos de calidad hacia las ciudades que los organizan contribuyendo a generar riqueza y creación de puestos de trabajo, muy necesarios en momentos de crisis como los que estamos padeciendo.

Culturales: la realización de un evento así, situaría el vino en una escala superior, y ya no se consideraría un simple acompañante en las comidas, sino un bien muy preciado. Este evento podría ser la pieza clave para promover estrategias de crecimiento a medio y largo plazo en Valladolid, y convertirla en una de las principales ciudades de destino turístico de España. El desarrollo de las acciones de enoturismo en la ciudad de Valladolid podría fomentar el turismo de la ciudad aumentando la demanda hotelera y de restaurantes ya que el perfil del que realiza enoturismo suelen ser familias con poder adquisitivo medio-alto, y que gastan entorno a 100 euros diarios durante los días que dura el acontecimiento.

\subsection{El enoturismo como pilar básico del Festival del Vino en Valladolid.}

Como símbolo duradero de la vida europea, el papel del vino ha venido evolucionando con el tiempo, cambiando desde una importante fuente nutritiva que era hasta convertirse en complemento cultural de la comida y de la convivialidad, compatible con un estilo de vida sano. El arte de la viticultura ha evolucionado, pero un principio que no ha cambiado para nada es la tradición Europea de presentar el vino y de comunicar sobre él mismo, centrándose en los orígenes, la herencia y la vinicultura.

Se suele asociar el vino con la gastronomía, la historia, la calidad de los productos locales y cierta solemnidad social. Como tal, a pesar de las diferencias en las pautas de consumo que se observan a través de toda la UE, el consumo moderado sigue siendo la norma general y es sólo una pequeña minoría la que hace un uso indebido del vino. Que el mundo del vino está cambiando es una realidad tangible hasta hace poco, el consumo general de vino estaba creciendo gracias a los mercados emergentes, sin embargo, la recesión ha deprimido el consumo total. El panorama vitivinícola español está siendo testigo de la llegada de grandes inversiones al sector en forma de infraestructura y tecnología con la construcción de numerosas bodegas (Del Rey, 2010:23).

El sector vitivinícola español es de gran importancia tanto por el valor económico que genera como por la población que ocupa. Entre las más bellas localidades rurales de España, un importante porcentaje pertenecen a lo que podría denominarse la España del vino. Algo similar puede afirmarse de los paisajes, y sobre todo, de las gentes que dedican sus vidas los viñedos. El consumidor español ha dado un salto cualitativo y, a 
pesar de que todavía queda mucho por hacer por la cultura del vino, la curiosidad, sobre todo del público joven, está ampliando el número de vinos que se consumen.

Actualmente, existe todo un mundo alrededor del vino, nuevas tendencias relacionadas con este sector, y la aparición de nuevos consumidores con gustos diferentes entre ellos, se habla de una cultura que caracteriza a los diferentes países en la que los vinos españoles se han abierto un hueco en los mercados internacionales, siendo muy valorada la relación calidad-precio. Los jóvenes que consumen vino en distintos países tienen bastantes más cosas en común que las generaciones que les precedieron, especialmente en los mercados occidentales. La llamada Generación Y o la Generación del "milenio" es menos individualista; a sus miembros les gusta sentir que forman parte de un grupo.

En España cabe reseñar dos grupos de consumidores bastante diferenciados por su edad y educación. En el primero hay una mayor proporción de jóvenes con un bajo conocimiento sobre el vino. Valoran que el vino proceda de una D. O. y su idoneidad con la comida con la que se vaya a acompañar. El otro segmento, está conformado por hombres adultos, con un bajo nivel de educación, pero con un nivel alto de conocimiento sobre el vino. Este segmento valora la etiqueta, la idoneidad del vino con la comida a la que acompaña y el haberlo probado anteriormente. La crisis económica ha producido un descenso del consumo de vinos a nivel mundial que afectó a los principales países productores, entre ellos España. Las denominaciones de Origen de Castilla y León con mayor volumen de comercialización no se libraron de esta disminución de las ventas en mercados extranjeros.

Valladolid se ha convertido en un referente entre las ciudades de vino gracias a su provincia, al ser la única que aglutina cinco denominaciones de origen. La ciudad ha conservado su memoria del vino presente desde su fundación. La calidad de los vinos de esta tierra es fruto del esfuerzo y entrega de las personas que trabajan en ello.

El enoturismo o turismo enológico tiene como objetivo potenciar y gestionar la riqueza vitivinícola de una determinada zona. Como consecuencia del creciente interés que suscita el enoturismo en la actualidad, la apuesta desde el sector se ha potenciado durante los últimos años convirtiéndose en una realidad.

En esta investigación se ha llevado a cabo para la recogida de información técnicas cualitativas. Se ha optado por el uso de éstas puesto que nos ofrece la oportunidad de explorar un tema del que no hemos realizado estudios previos. Por lo tanto, el análisis cualitativo proporcionará información que servirá como base para estudios cuantitativos posteriores. Dentro de las fuentes de información primaria se ha elegido la entrevista en profundidad.

\subsection{Zonas vitivinícolas con Imagen de Marca en el Mundo.}

El mundo del vino está cambiando, este hecho lo demuestran los datos publicados por la Oficina Internacional de la Viña y el Vino (OIV) que certifican como Europa pierde 
terreno respecto de una parte del Nuevo Mundo, Asia y Oceanía. Una tendencia ligera en superficie de viñedo, pero importante ya en términos de consumo y exportaciones (Muñiz, 2011:12).

La superficie total del viñedo mundial se ha mantenido casi constante en los últimos diez años. La única tendencia que resulta palpable es el ligero descenso de la superficie de viñedo en Europa compensada por el aumento en Asia, Oceanía y América tanto del Norte como del Sur.

Con los datos de 2001 España se mantiene a la cabeza de la superficie mundial de viñedo con 1.230 .000 hectáreas (15,53\% del total) seguida de Francia con 914.000 e Italia con 908.000. Detrás se encuentran países como Turquía (530.000), Estados Unidos (415.000), China (326.000), Irán (270.000), Portugal (261.000), Rumanía (247.000) o Argentina (205.000). Europa representa el 63\% del viñedo, seguida de Asia (19\%) y América (12\%).

El Valle de Napa (o Napa Valley) es una región de California en Estados Unidos, conocida principalmente por su producción de vino. El nombre Napa deriva de Wappo, que es el nombre de los indios que habitaron la región. El valle se localiza en el condado de Napa. Su longitud es de 48 kilómetros de largo, concentra más de 300 productores de vino y representa, de alguna manera, la nueva industria del vino, no sólo porque produce vinos de excelente calidad sino porque ha logrado la fusión con la gastronomía y el turismo. Cada año cerca de 8 millones de personas visitan el Valle de Napa, convirtiéndolo en una de las más populares atracciones turísticas de California. Una zona que ha sabido ver las posibilidades del Marketing Relacional contactando con los públicos de una forma muy acertada a través de las posibilidades de Internet (Alet, 1996).

Según comenta Paul Wagner (2008) a pesar de las dificultades económicas a nivel mundial, este mercado continúa creciendo gracias al aumento de la demanda de una nueva generación de consumidores que tienen mucho interés en vino, la Generación del Milenio. Se trata de personas de ambos sexos con edades comprendidas entre los 12 y los 32 años, que suponen más de 70 millones de la población estadounidense. Paul Wagner puntualiza que son jóvenes, no tienen ningún miedo y han nacido con un ordenador bajo el brazo y un MP3 en el bolsillo. No aceptan las tradiciones establecidas sobre vinos, son aventureros y para ellos el vino no es sólo una bebida, sino un billete para viajar a otros lugares, a otros países.

Paul Wagner en las Jornadas sobre el desarrollo socioeconómico (2008) señalaba que el enoturismo en sí no existe. Se trata tan sólo de un simple turismo. La gente no quiere estar todo el día catando vinos, por lo que hay que venderles calidad de vida, ocio, relax y diversión en partes iguales.

En cuestión de calidad de vino, muchas bodegas y vinos españoles tienen todas las cualidades para triunfar en el mercado americano. Sin embargo, muy pocas han hecho lo necesario para diferenciarse de las demás. Desde el punto de vista comercial y de 
marketing, el enfoque de venta de los vinos españoles necesita un cambio de estrategia. Desde hace años, todo se reduce a barricas, piedras, clima y fermentaciones. Por el contrario, el consumidor no tiene que ser enólogo para gozar del vino. El vino ha de comunicar la cultura de la región donde nace, ha de servir como símbolo de la música, poesía, arquitectura, la historia, la vida autentica de la gente que habita esa tierra, entonces ese vino se podrá vender (Wagner, 2008).

Hoy en día, Napa Valley es el hogar de cerca de 400 bodegas y numerosas marcas más. Sus productores y bodegueros combinan la ciencia de vanguardia con las técnicas tradicionales, y su reputación para la producción de vinos de calidad internacional está firmemente establecida en un mercado global cada vez mayor. Una comarca que goza de una gran imagen gracias a sus herramientas de comunicación internas y externas (Altés, 1993:78).

Entre otras, las diferentes actividades que se desarrollan en Napa Valley son las realizadas en El Napa Valley Wine, éste es un tren el cual ofrece un viaje relajante de tres horas a lo largo de treinta y seis millas de ida y vuelta entre la ciudad histórica de Napa y la pintoresca localidad de Saint Helena a través de uno de los valles más afamados del mundo del vino. Los huéspedes a bordo de El Wine Train disfrutarán de la opción de una comida o cena preparada a la orden, mientras que ven los viñedos y bodegas abordo de los vagones Pullman de la época 1915-1917, o el vagón Vista Dome de 1956 que ha sido restaurado espléndidamente. La cata de vino en el Valle de Napa puede ser desalentadora, hay cientos de bodegas para visitar. Si planea comprar vino, hay miles para elegir. Pero ¿cómo sabe que bodegas visitar?, es difícil saberlo. El grupo de profesionales del vino a bordo del tren le podrían informar a cerca de las diferentes ofertas existentes (Diccionario del Vino, 2011).

La región de Burdeos de Francia es la segunda región vinícola más grande del mundo, con 284.320 acres de vid. Esta ciudad francesa, vieja dama aristocrática del vino, continúa siendo un destino obligado para los amantes del vino y la gastronomía. Su arquitectura clásica y su ópera son alguno de sus atractivos a los que se suman nuevas zonas comerciales y de restaurantes en lo que era el antiguo puerto que completan la oferta de la sexta ciudad francesa por tamaño.

En Burdeos el concepto de terroir tiene un papel trascendental en la producción, con las fincas destacadas luchando por hacer vinos marcados por el terroir que reflejan el lugar del que vienen a menudo de uvas recogidas en un solo viñedo. El suelo de Burdeos está compuesto por grava, piedra arenisca, y arcilla. Los mejores viñedos de la región están ubicados en los suelos de grava bien drenados que se encuentran frecuentemente cerca del río Gironda. Un viejo adagio en Burdeos es que las mejores fincas pueden "ver el río" desde su viñedo y la mayor parte de tierra que enfoca la ribera del río están ocupadas por fincas clasificadas.

En el norte, el Médoc es un sitio privilegiado para la producción del vino tinto: es aquí donde se producen los muy conocidos Pauillac, Margaux, Listrac, Moulis, Saint-Julien y Saint-Estèphe. Al Sur de Burdeos se producen los vinos de tipo graves (Pessac, 
Léognan) y los vinos generosos. Las fincas donde se producen esos tan célebres y generosos (Cerons, Sauternes, Barsac en la orilla izquierda del Garona y Sainte-Croixdu-Mont, y Loupiac en la orilla derecha), se sitúan en un enclave localizado en la punta Sur de los "graves", cerca de Langon. Sémillon y Sauvignon son las dos cepas principalmente cultivadas para producir los vinos blancos, y están combinadas con una geología comparable con la del alto Médoc que se compone de pedregal, arena, arcilla y marga y cuyo clima es generalmente cálido y soleado (Guía de vinos Burdeos, 2011).

La Celebración de la fiesta del vino en Burdeos es una de las mayores festividades que tiene lugar en Europa y la iniciativa del Ayuntamiento de Burdeos (Francia), es una celebración que incluye lo mejor de la gastronomía y la cultura y que tiene lugar a orillas del Garona en la mayor Plaza de Europa. La fiesta se extiende durante 4 días en el corazón mismo de la ciudad de Burdeos en una superficie de 12 hectáreas y se llama Bordeaux fete le vin, realizándose del 24 al 27 de junio. Se trata de un evento que está dirigido a todo turista o residente que sea amante del vino, de su sabor, de su olor o a cualquiera que quiera acudir a este acontecimiento. Esta es una festividad que incluye degustaciones y paseos por los viñedos de Burdeos y de su región.

\subsection{El Vino en España.}

El panorama vinícola español está siendo testigo de la llegada de grandes inversiones al sector en forma de infraestructura y tecnología con la construcción de numerosas bodegas (Campo, 2009).

No obstante el vino español padece muchos y peligrosos inconvenientes: cae el consumo, pierde popularidad al ser considerado como una bebida alcohólica, se ve penalizado por el precio que adquiere en los restaurantes, se estancan las exportaciones y reconocen los propios productores que no saben venderse bien. Pero lo que hace que todo lo anterior sea sumamente grave es una tremenda sobreproducción con millones de litros que no se mueven (Alonso, 2009).

El sector vitivinícola español es de gran importancia tanto por el valor económico que genera como por la población que ocupa. Entre las más bellas localidades rurales de España, un importante porcentaje pertenecen a lo que podría denominarse la España del vino. Algo similar puede afirmarse de los paisajes, y sobre todo, de las gentes que dedican sus vidas los viñedos y las bodegas. Puede decirse que no hay en España, a diferencia de la arquitectura románica, las corridas de toros o la producción de aceite de Oliva, Comunidad sin vino. La proliferación de bares y tabernas con vinos por copas, la ampliación de las cartas de vinos de algunos restaurantes, el aumento del consumo de vino con denominación de origen, el boom de la gastronomía y su estrecha relación con el vino, son sólo una muestra de los cambios que han experimentado los hábitos de los españoles a la hora de tomar vino (Junta de Castilla y León, 2011). 
No obstante, es de destacar que el consumidor español ha dado un salto cualitativo y, a pesar de que todavía queda mucho por hacer por la cultura del vino, la curiosidad, sobre todo del público joven, está ampliando el número de vinos que se consumen. Así, por ejemplo, el consumo de vinos con denominación de origen subió casi un litro y se situó en 8,2 litros per cápita, mientras que el de vino de mesa bajó ligeramente a 18,2 litros en comparación con los 18,8 litros de 2003. Desde hace varios años, las estadísticas muestran este salto cualitativo, que también se refleja en la diversidad de vinos consumidos. Ya no sólo se oye hablar de los clásicos, en boca de todos, como Rioja o Ribera del Duero. El vino consumido fuera del hogar sube, ya que con casi el $60 \%$ en bares, cafeterías, hoteles, restaurantes e instituciones, este segmento absorbió 714,44 millones de litros, lo que representó el 9,2\% más. El restante 40,4\% del vino consumido en el mercado interno correspondió a los hogares españoles, con 483,35 millones de litros, cifra que, por el contrario, supuso un retroceso del 3,9\% en comparación con el año 2003 (Cámara de Comercio de Valladolid, 2011).

Álvaro Cerrada (2010), sostiene que ni un 20 por ciento de las bodegas españolas utilizan las redes sociales para interactuar con el cliente, desarrollar acciones de promoción y conseguir aumentar sus ventas. Alude a los más de diez millones de personas que en España tienen cuenta abierta en Facebook, cifra muy alta para la escasa presencia del marketing de vino en esta herramienta. YouTube, segundo canal de Internet más utilizado después de Google, precisa este especialista del marketing, es otra red social también muy cautiva en el sector del vino y muy poco utilizada por las bodegas.

\subsection{Actividades enológicas más destacas en Castilla y León.}

Se sabe que Castilla y León es una de las comunidades más desarrolladas en temas relacionados con el vino, tanto es así, que en el año 2010 se creó un canal de vinos en esta comunidad, donde van apareciendo las diferentes actividades que se van desarrollando, así como, numerosas herramientas para que las personas interesadas puedan interactuar con este canal. No se ha de olvidar que el contacto a través de Internet es fundamental al igual que cuidar el sitio web de la bodega o empresa relacionada con el enoturismo (Gómez, 2006).

Este canal nace con la intención de convertirse en un portal de referencia sobre los vinos de Castilla y León. El objetivo de este proyecto es acercar a la sociedad la cultura del vino y fomentar su consumo, a través de tres pilares fundamentales. Por un lado, se pretende ofrecer más información para transmitir la realidad del sector; por otro lado, se organizarán eventos relacionados con el vino, tanto dentro como fuera de Castilla y León; gracias a un nuevo vehículo de comunicación, el canal www.castillayleondevinos.com (Diputación Provincial de Valladolid, 2011).

La crisis económica ha producido un descenso del consumo de vinos a nivel mundial que afectó a los principales países productores, entre ellos España. Las Denominaciones de Origen de Castilla y León con mayor volumen de comercialización no se libraron de esta disminución de las ventas en mercados 
extranjeros. Es por tanto, fundamental desarrollar buenos canales de comercialización y comunicación para favores la venta y la mejora de la imagen corporativa. En este sentido, Internet y la era digital tiene mucho que decir (Burgos y León, 2001: 89).

Rafael del Rey, sostiene que las denominaciones de origen (D.O) e indicaciones geográficas protegidas son un elemento extraordinario para potenciar el consumo de vino, los vinos de calidad y promocionarlos.

Un total de 1,3 millones de botellas fueron comercializadas en 2011 fuera de España, de las que más de un millón fueron de tintos de reserva, lo que permitió mantener la cifra total de ventas ligeramente por encima de 3,83 millones de botellas, muy parecida a la de 2010, compensando así el fuerte descenso en el mercado nacional (Diputación Provincia de Valladolid, 2011).

Situando a la ciudad de Valladolid en el marco de Castilla y León, ésta se ha convertido en un referente entre las ciudades del vino gracias a su provincia, al ser la única que aglutina cinco Denominaciones de Origen. La ciudad ha conservado su memoria del vino presente desde su fundación. Muchos de los Monasterios que promueven la cultura vitivinícola se han mantenido hasta hoy en día gracias a que la mayoría de las bodegas que existen vertebran su existencia en las posadas y tabernas de la antigüedad (LLorca, 2009).

La provincia de Valladolid tiene muchas características que la distinguen: la horizontalidad de la Tierra de Campos; los verdes campos en Tierra de Pinares; las historias de los Montes Torozos...Es una provincia diferente, diferenciada de norte a sur de Este a Oeste, pero en sus $8.000 \mathrm{kms}$ de extensión hay un elemento en común, la vid. Valladolid es la única provincia de España que cuenta con cinco denominaciones de origen: D. O. Rueda; D. O Ribera de Duero; D. O. Cigales; D. O. Toro y D. O. Vinos de Tierra de León (Ayuntamiento de Valladolid, 2011).

La calidad de los vinos de esta tierra es fruto del buen hacer de las personas que trabajan en ello, cuidando con esmero todo lo que tiene que ver con el vino. El enoturismo o turismo enológico tiene como objetivo potenciar y gestionar la riqueza vitivinícola de una determinada zona, en este caso la provincia de Valladolid, por todo ello, la Institución Provincial, en el año 1999, decidió apostar fuerte por la creación del Museo Provincial del Vino en el castillo de Peñafiel.

Este museo cumple también el objetivo de promocionar el conocimiento a través del mundo del vino, de la provincia de Valladolid, de sus aspectos culturales y geográficos. Este museo cuenta con una importante variedad de actividades que se adaptan a los diferentes intereses del público: catas dirigidas, cursos de catas para profesionales o para los que empiezan que quieran iniciarse durante un fin de semana. Incluso se ofertan divertidos talleres para los escolares como "El juego de los sentidos". La filosofía de este proyecto, contempla el diálogo con otras regiones vitivinícolas del mundo: Vinus Mundi es un programa abierto que nos permite 
asomarnos a ellas a través de exposiciones, catas dirigidas, conciertos etc.... (Guía del Vino Anaya, 2007).

Valladolid es una ciudad, que con el paso del tiempo se ha convertido en una de las ciudades de referencia del vino a nivel nacional en esta área. El pasado 2011, acogió una de las ferias más importantes del vino de nivel mundial: Iberwine.

Esta feria del vino español, portugués y latinoamericano tuvo lugar del 25 al 27 de mayo en el recinto ferial de la ciudad. Fue un evento sólo para profesionales de la industria del vino. Con este encuentro se pretende también promover los negocios para desarrollar una nueva conciencia de la industria del vino para su posterior desarrollo en todos los frentes: económico, social y medio-ambiental. Además, Iberwine 2011 quiere ir aún más allá, y por eso, en su nueva concepción fusiona el vino, los negocios, la cultura y el arte para crear un novedoso evento de sensaciones dentro y fuera del recinto ferial con áreas comerciales, áreas Vips, área de descanso...

En Febrero de 2011 tuvo lugar el Concurso Vino de Museo 2011, convocado por el presidente de la Diputación de Valladolid. En dicho concurso podrán participar todas las bodegas de la Comunidad de Castilla y León siempre que estén adscritas a una de las Denominaciones de Origen de la región o bajo la figura de Calidad de Vino de la Tierra de Castilla y León. Tuvo lugar en la sede del Museo Provincial del Vino en el Castillo de Peñafiel. El director técnico de cata fue el presidente de los sumilleres de Castilla y León, Pablo Martín afirmando que la labor de un sumiller es ayudar al consumidor a elegir el vino adecuado con el plato y contribuir a descubrir el amplio mundo de sabores y olores con que puede disfrutarse de una copa de vino (Norte de Castilla electrónico, 2011).

Valladolid cuenta con una situación privilegiada en el panorama enoturístico nacional, gracias a las cinco denominaciones de origen vitivinícolas con las que cuenta. Ahora mismo censadas en toda la provincia hay un total de 374 bodegas, de las cuales, 60, son visitables, y ofrecen sus instalaciones para el desarrollo de todo tipo de actividades. Algunas bodegas, ofrecen ya la posibilidad de organizar eventos de hasta 1.500 personas, en unos entornos únicos y originales, y pudiendo desarrollar un sin fin de actividades tanto para grandes como para pequeños grupos. En cada una de sus zonas vitivinícolas existen multitud de bodegas, desde las históricas a las más vanguardistas, restaurantes, alojamientos que ofrecen estancias especiales, tiendas de vino, museos, artesanía, fiestas y tradiciones, catas diferentes... Una variada oferta que despierta el interés del viajero acercándole al interesante mundo del vino (Norte de Castilla electrónico, 2011).

La oficina de Enoturismo de Valladolid creada en 2004, se ha implantado para promocionar y comercializar los vinos de la provincia, en colaboración con las principales empresas del sector, bodegas, alojamientos y municipios, pretende organizar, coordinar y aunar los esfuerzos de un sector en auge, ofreciendo al visitante nuevas formas de entender y comprender la tradición y la oferta relacionada con el 
mundo del vino. La oficina de enoturismo ofrece diferentes alternativas para visitar las bodegas de Valladolid:

Rutas de un día o de fin de semana por cada una de las denominaciones de origen que nos recorren.

Ruta circular para visitar de forma completa las cuatro zonas vinícolas y un programa semanal para los más apasionados por los vinos de alta expresión. En definitiva, un turismo de calidad, ofreciendo visitas guiadas garantizadas, visitas en idiomas, catas dirigidas y venta de productos de cada zona.

Rutas que combinan todos los tipos de recursos existentes en cada una de las zonas. Rutas por cada una de las Denominaciones de origen que recorren la provincia, Ribera del Duero, Rueda, Cigales, Toro y Tierras de León.

Ruta Urbana enogastronómica en la que se adentra en el Valladolid histórico, combinando lo mejor del Patrimonio cultural de la ciudad con las zonas de vinos y pinchos, todo ello relacionado con el esplendoroso pasado vitivinícola que vivió la ciudad en la época de Felipe II.

La oficina de turismo con la colaboración de la Diputación de Valladolid ha organizado El Bus del Vino que viaja por las carreteras de la provincia de Valladolid con destino a la Ribera del Duero, esta propuesta une el arte, el patrimonio artístico, la gastronomía y el vino que recorre los municipios más representativos del territorio vallisoletano. Hay que destacar la importancia que tiene el Museo de la Ciencia en los eventos enológicos, ya que, en ocasiones se celebran catas, exposiciones, ponencias etc.... Este museo abrió sus puertas el 29 de abril de 2003. Es un museo creado por iniciativa municipal con la intención de que los residentes y los visitantes de la ciudad disfruten del apasionante mundo de la Ciencia (Norte de Castilla electrónico, 2011).

\subsection{Campañas Promocionales de Enoturismo en Valladolid.}

De entre las campañas más destacadas para promocionar los vinos de la provincia de Valladolid, tenemos que hacer referencia a la que tuvo lugar el 8 de Marzo de 2011 en Miami. El acto central de la campaña Drink Ribera, Drink Spain fue protagonizado por 123 bodegas y 324 vinos. El objetivo principal de esta campaña era que los vinos de Ribera de Duero tuvieran más presencia en el estado de Florida.

El Grand Tasting de Miami se reforzó con otras acciones en vinotecas y establecimientos destacados del Estado de Florida, catas y presentaciones que impartieron el equipo del Consejo Regulador D.O. Ribera del Duero en EE UU.

El Salón de la Alimentación en Valladolid apostó en 2011 por un concepto ferial innovador y profesional, adaptado a los modelos de negocio actual y respaldado con la experiencia de doce ediciones. Una de las características que definen este Salón de la Alimentación 2011 es su carácter exclusivamente profesional, durante sus tres jornadas 
realiza un programa de catas en el que se divulgan productos amparados por sellos de calidad como Denominaciones de Origen, marcas de garantía o indicaciones geográficas protegidas. El objetivo de estas catas es dar a conocer a restauradores, hosteleros y compradores en general sobre las características de los productos que protagonizarán estas sesiones. Parejas de expertos formadas por un sumiller y un miembro de la Academia Gastronómica realizaron maridajes en los stands de los diferentes expositores con productos y vinos expuestos en el Salón.

\section{METODOLOGÍA}

Entre los principales objetivos de la investigación se destacan los siguientes:

-Saber si la ciudad de Valladolid podría acoger un importante evento de enoturismo a grandes niveles.

-Conocer las características de los nuevos consumidores de vino y actividades de enoturismo en la comarca.

-Entender la importancia del enoturismo para el desarrollo de la provincia de Valladolid.

Las fuentes con las que se ha contado para llevar a cabo esta investigación han sido de dos tipos, primarias y secundarias, a través de las cuales se han obtenido un amplio marco contextual que avala y nutre las hipótesis propuestas en la investigación.

Fuentes de información primaria: se entiende por fuente primaria aquella que ha sido elaborada de primera mano y de manera específica por el investigador para satisfacer las necesidades de su estudio. Las utilizadas para el diseño de este artículo han sido las entrevistas en profundidad a profesionales del sector enoturístico y partiendo de esta definición, se entiende que el diseño de la entrevista es una fuente primaria de información, así como el análisis de los datos de la misma, la cual ha sido expuesta y comentada al final del artículo.

Fuentes de información secundaria: se entiende por fuente de información secundaria aquella documentación ya elaborada con anterioridad, con fines distintos, o no, a los del propio estudio, a los que el investigador acude para contrastar o respaldar los planteamientos de su investigación. Este tipo de fuentes han sido imprescindibles para la elaboración teórica de la introducción y práctica del estudio. Así pues, las fuentes de información secundarias a las que se ha recurrido han sido principalmente las siguientes:

- $\quad$ Páginas Web.

- $\quad$ Artículos especializados.

- $\quad$ Revistas especializadas de vino.

- Periódicos relacionados con el tema en cuestión.

- Información sobre conferencias, ponencias etc....

- Bibliografía

- $\quad$ Recursos audiovisuales: videos, documentales...

- Recursos electrónicos: páginas Webs sobre turismo enológico en Valladolid, Web oficial de la oficina de congresos...

- Informes, boletines turísticos sobre enología... 
Finalmente, se han redactado las valoraciones y conclusiones de la investigación, así como nuevas propuestas de investigación en la misma línea para poder realizar en un futuro. No obstante, la presente investigación parte de un estudio previo sobre la comparativa de contenidos enoturísticos de la D.O. Ribera del Duero y la D.O.C. Rioja en la Web.

A continuación se presentan las hipótesis que mediante la realización de la investigación, se comprobará si se cumplen o se refutan finalmente:

1. Valladolid se concibe como capital idónea de la comunidad de Castilla y León para desarrollar el festival del vino.

2. Los nuevos grupos de consumo de vino han creado nuevos estereotipos en la sociedad, que crean otras tendencias destinadas especialmente a este sector.

Respecto a la primera hipótesis planteada, se han fijado las siguientes preguntas de investigación:

1. ¿Es Valladolid la ciudad idónea para la realización de este evento?

2. ¿Qué repercusión tendría para Valladolid y su entorno un evento de estas características? Estaríamos hablando de repercusiones de tipo social, económica o cultural.

3. ¿Cuáles son las motivaciones para realizar un evento de esta envergadura?

4. ¿Consideras que la promoción de Valladolid en relación a su oferta enológica es adecuada? ¿En qué lo mejoraría?

Respecto a la segunda hipótesis planteada, se han fijado las siguientes preguntas de investigación:

5. ¿Cuáles son las tendencias que empiezan a producirse y que continuarán en un futuro?

6. ¿Los cambios de las actividades relacionadas con el vino reflejan una nueva percepción de éste en la sociedad?

7. ¿A los nuevos consumidores de vino se les puedes catalogar de nuevos estereotipos de consumo en la sociedad?

\section{ANÁLISIS Y DISCUSIÓN}

La entrevista en profundidad según apunta Cervera Fantoni (2006:69), es una técnica cualitativa para la indagación y recolección de datos basada en el procedimiento de interrogación y mediante la observación directa no participante. La entrevista en profundidad facilita datos que se extraen de un informante y que tienen que ver con el objeto de la investigación. Es una entrevista personal no estructurada en la que se persigue, de forma individual, que cada entrevistado exprese libremente sus opiniones y creencias sobre algún tema objeto de análisis, es un proceso de interacción dinámica de comunicación entre dos personas, entrevistador y entrevistado, bajo el control del primero. Generalmente se usan cuando se requiere conseguir información muy compleja, delicada y nueva en el campo de investigación. 
Ventajas de la entrevista en profundidad:

Es una técnica muy útil cuando se trata de analizar temas que exigen un gran anonimato. Permite conocer en profundidad los temas y obtener información muy rica.

Es una técnica muy válida cuando tenemos muy poca información sobre un tema y queremos hacer una investigación explorativa.

Desventajas de la entrevista en profundidad:

El entrevistado/a puede tener problemas para manifestar sus puntos de vista respecto de un tema concreto. La gente puede tener dificultades para recordar y expresar detalles, datos y concreciones sobre un tema.

El investigador/a está bajo el poder del entrevistado/a; depende completamente de él.

\subsection{Desarrollo y registro de la información en la Entrevista en Profundidad.}

Las entrevistas de este tipo exigen la concentración tanto del entrevistador/a como del entrevistado/a. Esto supone obstaculizar lo menos posible la concentración del entrevistado/a cuando se recoge o registra la información. Teniendo en cuenta este aspecto, se ha elegido un buen sistema para grabar la información. Por otra parte se han llevado a cabo en diferentes salas de los puestos de trabajo de los entrevistados.

La técnica utilizada para registrar la información de cada una de las entrevistas realizadas ha sido la grabación. Se ha pedido cita previa, y ninguno de ellos ha puesto ningún impedimento para registrar la información a través de una grabadora de voz.

Las entrevistas han sido realizadas a personas con gran experiencia relacionadas con el mundo del vino y de eventos de la ciudad de Valladolid. Se ha intentado conocer distintos puntos de vista, para tener una visión más amplia del tema planteado. Se ha entrevistado a Rodrigo Criado del rey, Carolina Toribio de Benito y Gustavo Calvo Díez.

La decisión de haber seleccionado estas personas para las entrevistas ha sido fundamentalmente por la relación que tienen con temas enológicos y la implicación directa con las actividades relacionadas. A continuación se exponen las personas seleccionadas para las entrevistas y su correspondiente justificación:

Rodrigo Criado del Rey Saéz

Coordinador de eventos y turismo en la Oficina de Congresos de Valladolid

Se ha decidido realizarle la entrevista por sus amplios conocimientos en organización de eventos y congresos en Valladolid. Además es la persona que nos puede comentar 
los puntos débiles y fuertes de la ciudad, así como destacar las actividades promociónales que se están llevando a cabo.

Carolina Toribio de Benito

Coordinadora de enoturismo y gastronomía en Valladolid

La decisión de hacerle la entrevista ha sido fundamentalmente por los conocimientos de enología centrados en la ciudad de Valladolid. Nos ha proporcionado una visión más técnica, global y precisa del tema.

\section{Gustavo Calvo Díez}

Responsable de la boutique de maridaje de vino, Señorita Malauva en Valladolid.

La elección de Gustavo para la realización de la entrevista ha sido principalmente para aportarnos una visión más comercial y más cercana de la situación actual de la ciudad. Ha trabajado en bodegas organizando eventos y en al actualidad es propietario de una tienda vitivinícola donde se realizan varias actividades enoturísticas.

\subsection{Análisis de la información obtenida.}

\section{RODRIGO CRIADO DEL REY SÁEZ}

Coordinador de eventos y turismo en la Oficina de Congresos de Valladolid

Desde un primer momento Rodrigo Criado del Rey considera que Valladolid reúne las condiciones adecuadas para que un evento de esta magnitud y temática se pueda realizar. La ciudad y su provincia poseen cinco denominaciones de origen de vino y ninguna más en España cuenta con estas características enoturísticas, ni siquiera la D.O.C. Rioja.

Todas las repercusiones de un evento de esta magnitud serían positivas. Desde el punto de vista social, entre otras ventajas, supondría que un gran número de vallisoletanos tuvieran un conocimiento muy elevado de todo lo relacionado con el vino dado que Valladolid es una ciudad puntera en este aspecto.

Se está promocionando a gran escala todo lo referente a la enología y al enoturismo económico. Es evidente que traer un evento a Valladolid generaría un movimiento hotelero, comercial, cultural etc., muy importante. Cualquier tipo de evento que fomente la tradición de la enología en Valladolid y su provincia reforzaría esa cultura del vino.

La tendencia actual que se ha despertado en Valladolid desde hace unos años con todo lo que rodea al vino, ha sido muy novedosa. Antes, simplemente se producía y vendía, ahora la tendencia es introducirse en esas bodegas, ver cómo se hace el vino, cómo se 
recolectan las uvas, enseñar su arquitectura, etc. Esta explosión del enoturismo y de la apertura de las bodegas de vino al público, ha traído consigo que aumente el número de consumidores.

La promoción que realiza Valladolid sobre su oferta enológica a todos los niveles es adecuada, lógicamente se podrían hacer un mayor número de actividades, pero en este momento con la crisis actual no se pueden emplear más recursos. Comenta que se pueden desarrollar más y mejores acciones, entre otras, viajes de familiarización de prensa especializada en enoturismo, visitas culturales etc. todo ello con el objetivo final de atraer y dar a conocer el potencial de Valladolid.

Por otra parte, la ciudad posee cinco denominaciones de origen muy importantes, una de ellas, D.O. Ribera del Duero, mundialmente conocida y la joya de la corona de las denominaciones de origen. Rodrigo Criado comenta que se podría promocionar más, por ejemplo, con un mayor acercamiento de periodistas especializados para enseñarles in situ la oferta con la que cuenta para que ellos a su vez lo divulguen a través de los distintos medios de comunicación.

\section{CAROLINA TORIBIO DE BENITO}

Coordinadora de enoturismo y gastronomía en Valladolid

Según Carolina Toribio Valladolid es una ciudad muy adecuada para realizar el festival de vino ya que posee un gran potencial con cinco denominaciones de origen muy reconocidas a nivel nacional e internacional, además de ese potencial, posee un gran atractivo turístico, cultural, gastronómico etc. Sería muy beneficioso aunar todo este potencial y crear un macro evento.

Siempre han existido las fiestas de la vendimia porque tienen una gran tradición en la provincia y en muchas localidades de ésta se siguen celebrando como en Cigales, Peñafiel etc.

Carolina Toribio comenta que habría que unificar de alguna forma todo este conglomerado de ideas, y opina que aparte de organizar una fiesta de la vendimia específica, también se podrían hacer un sinfín de actividades relacionadas con el mundo del vino para fomentar su cultura entre la gente. El sitio idóneo y donde más calidad habría para llevarlo a cabo sería en Valladolid, por ser el centro neurálgico de la provincia.

La repercusión que puede tener para Valladolid un evento de este tipo seria muy importante. Desde su punto de vista, considera que no debe ser un evento único y aislado en un año concreto, sino, como una actividad fundamental para incluirla dentro de todas las actividades o de toda la oferta cultural que pueda haber en una ciudad. 
Los beneficios que puede aportar y su repercusión a todos los niveles, social económico y cultural, podrían convertirse en un gran atractivo para dar a conocer, y a atraer un importante turismo económico y cultural, ya que no dejará de ser una actividad global.

Respecto a las motivaciones para realizar un evento de estas características, Carolina Toribio explica que no hay nada similar. Añade que se están estudiando este tipo de eventos o similares llevados a cabo en otros países, como en Argentina donde la fiesta de la vendimia es una fiesta de interés nacional que atrae a multitud de público de todo el mundo.

El festival del vino que realizan en Burdeos es espectacular, en todos los eventos que realizan involucran a todos los habitantes de la ciudad y alrededores, celebran el festival del vino con un sin fin de actividades relacionadas con este mundo. La motivación pasa por implicar al mayor número de personas para que tomen conciencia de lo que supone la organización de un evento de estas dimensiones. Carolina Toribio afirma que no se cuenta con nada que se asemeje a la colaboración ciudadana, pero sí potencial para poder hacerlo.

En cuanto al consumo, la tendencia actual ha variado bastante con respecto a años anteriores, algunos estudios indican que su consumo se ha reducido. Comenta que ya no se bebe el vino como en la época de nuestros abuelos que lo hacían a granel. En torno al vino y en la actualidad representa algo más, existe una cultura relacionada con él y su público Cada vez se está más interesado en conocer y profundizar en todo lo que respecta a este tema.

Para atraer a los jóvenes a consumir vino de forma moderada se deben realizar actividades dirigidas a ellos, con múltiples acciones atractivas, para que este público tome contacto y empiece a disfrutar de su consumo.

Actualmente, se realizan muchas más actividades relacionadas con el mundo del vino. Hoy día, se pueden encontrar empresas que realicen catas, otras relacionadas con el enotursmo, etc. Se ha producido un aumento espectacular de tiendas especializadas que organizan actividades relacionadas con el mundo del vino como juegos, cursos de inglés, etc.

En realidad, quizá hoy día mucha gente se introduce en el mundo del vino por estar de moda, o también porque están interesados en tener conocimientos nuevos. La sociedad actual en la que vivimos es un público más inquieto y deseoso de conocer todo lo relacionado con la cultura del vino.

Respecto a la promoción que realiza Valladolid a cerca de su oferta enológica, Carolina comenta que en los últimos años se "están haciendo las cosas bien", pero queda "mucho camino por andar" y se está trabajando de forma insuficiente. En Valladolid se debería centrar más la promoción no sólo en lo relacionado con el vino, si no también en otros productos turísticos muy importantes: congresos, ferias, rutas 
culturales, la lengua etc. Todos ellos se podrían complementar con el tema del enoturismo y sería muy importante unificar criterios, para que todos los organismos implicados, ya sean públicos o privados estén integrados.

\section{GUSTAVO CALVO DÍEZ}

Responsable de la boutique de maridaje de vino, Señorita Malauva

El responsable de la boutique de maridaje de vino, opina que Valladolid es una de las provincias con más denominaciones de origen. Tanto los propios habitantes de la ciudad como de la provincia no conocen suficientemente las capacidades que tiene a la hora de elaborar vinos. Cuenta con cinco denominaciones de origen, Ribera de Duero, Rueda, Toro, Cigales y Tierra de León, que son muy importantes.

Valladolid es una ciudad que está "rodeada por el vino", con restaurantes, tiendas especializadas en vino, museos etc. en su opinión, afirma que se puede hacer un gran evento dada su potencialidad y prestigio. Piensa, que es la mejor publicidad para la ciudad.

Un evento de estas características afecta a todos los ámbitos, desde el punto de vista económico repercute en el aumento del número de restaurantes, de bares, de tiendas, hoteles etc.

A nivel social incide en el propio público de la ciudad que se le educa de cierta forma para conocer qué tipos de vinos existen, elevar su conocimiento, y tenga como estandarte a Valladolid, una ciudad pionera en todo lo relacionado con el vino.

A nivel cultural, puede ir asociado a exposiciones, conciertos y a todo tipo de espectáculos y actividades para animar al público a participar en el evento y que elijan la ciudad como destino turístico.

Según explicaba Gusta Calvo, hay muchos motivos para la realización de un evento de esta magnitud. En Valladolid, hay pocas posibilidades de turismo teniendo en cuenta que la ciudad cuenta con un considerable patrimonio histórico-cultural, pero está desorganizado. El motivo principal de la realización de este festival es llegar al mayor número posible de público para que Valladolid sea una ciudad de visita obligada y de reclamo turístico por su gastronomía y su vino, eso es lo más importante a la hora de "vender" Valladolid como destino enoturístico.

Gustavo Calvo afirma que el consumo de vino ahora se ha reducido, hemos pasado de una generación a otra. El vino antiguamente era una cuestión de señores y ahora es una cuestión de varias generaciones. Afirma que su tienda es muy dinámica y divertida, plantea el consumo del vino no como un producto que beben unos señores con bigote y puro sino enfocado a la gente joven. 
Todas las actividades que realizan, están orientadas a este tipo de público y, lo que cree es que el vino junto con el diseño de etiquetas y el cambio de imagen tiene que ir evolucionando para las generaciones jóvenes que van a consumirlo.

Gustavo afirma con contundencia que los cambios de las actividades del vino reflejan una nueva percepción de éste en la sociedad, ya que el vino en épocas anteriores era un producto que nuestros mayores lo utilizaban como alimento, una combinación de pan y vino, para reponer energías, para trabajar, para ciertas celebraciones etc. Ahora el vino va mucho más allá, es un producto de diseño, de consumo habitual, de relaciones sociales, etc. y al final lo que ha generado, son otros negocios a su alrededor, "marketing experiencial", turismo de experiencia, la creación de bodegas donde hay hoteles, spa y todo tipo de actividades con la creación del enoturismo urbano.

Este joven emprendedor considera que desde el punto de vista de la promoción turística se está haciendo francamente bien. Se está potenciando Valladolid como un destino de vino, de gastronomía y de hecho con el fin de atraer público de todas las edades, haciendo varias actividades al año, entre ellas el concurso de pinchos. Considera que desde el punto de vista profesional la promoción que realiza Valladolid es mejorable fundamentalmente de información al cliente: en los restaurantes y bares no hay buenos profesionales, no hay buenos sumilleres, tampoco profesionales que informen correctamente de las posibilidades de Valladolid, no existe personal suficientemente formado para atender correctamente a sus clientes.

El público que viene de fuera trae la idea de que hay una oferta muy variada de vinos y después no la encuentran realmente porque quizá no se les atiende correctamente. En la mayoría de los bares están expuestos los mismos vinos, esto hace que el público no tenga una variedad ni oferta a la hora de escoger.

El hacer ferias y otro tipo de actividades supone desarrollar el potencial enológico con el que cuenta Valladolid, pero si después la imagen que se lleva el cliente o turista que viene a la ciudad no es la que debiera ser, por las razones expuestas, sería contraproducente.

\subsection{Interpretación y discusión de la información obtenida en las entrevistas.}

Todos los entrevistados consideran Valladolid como la ciudad idónea para la realización de este festival de vino, ya que, cuenta tanto la ciudad como su provincia con cinco denominaciones de origen reconocidas a nivel nacional e internacional. Además de ese potencial, posee un gran atractivo turístico, cultural, gastronómico etc. Tanto los propios habitantes de la ciudad como de la provincia no conocen suficientemente las capacidades que tiene a la hora de elaborar vinos, por ello un evento de estas características podría dar a conocer este potencial. Valladolid tiene mucha experiencia en el sector, es decir, cuenta con una oficina de enoturismo, que desarrolla actividades en otras provincias de la comunidad de Castilla y León. 
En cuanto a infraestructuras, urbanísticamente la ciudad de Valladolid tiene una gran oferta de plazas hoteleras además de una excelente red de comunicaciones tanto por tierra como aire que podría favorecer en gran medida su desarrollo. Todas las repercusiones de un evento de esta magnitud serían positivas.

Carolina Toribio añade que habría que unificar de alguna forma todo este conglomerado de ideas. El sitio idóneo y donde más calidad habría para llevarlo a cabo sería en Valladolid por ser el centro neurálgico de la provincia, añade Gustavo. A nivel social incide en el propio público de la ciudad que se le educa de cierta forma para conocer qué tipos de vinos existen y elevar su conocimiento en esta materia. A nivel cultural, puede ir asociado a exposiciones, conciertos y a todo tipo de espectáculos y actividades para animar al público a participar en el evento y que elijan esta ciudad como destino turístico con todas las ventajas que supone a todos los niveles.

Respecto a las motivaciones para realizar un evento de estas características, Carolina Toribio comenta que todavía no se cuenta con nada parecido. Añade que se están estudiando otro tipo de eventos llevados a cabo en otros países y se debería aprovechar esa experiencia trasladarla aquí.

Gustavo Calvo afirma con contundencia que hay muchos motivos para la realización de un evento de esta magnitud. El motivo principal de la realización de este festival es llegar al mayor número posible de público para que Valladolid sea una ciudad de visita obligada y de reclamo turístico por su gastronomía y sus excelentes vinos. Lo que se intenta con estas acciones es atraer, no solamente a los visitantes expertos o amantes del vino, sino a los periodistas más reconocidos tanto a nivel nacional como internacional, para que conozcan tanto la ciudad de Valladolid como al resto de provincias de Castilla y León y conformen una opinión positiva de las calidades del vino, disfruten de la estancia y desarrollen y difundan en sus diferentes medios de comunicación las potencialidades de la comunidad, añade.

Gustavo añade que los cambios de las actividades del vino reflejan una nueva percepción de éste en la sociedad, ya que el vino en épocas anteriores era un producto que nuestros mayores lo utilizaban como alimento. Según Gustavo la tendencia actual que se ha despertado en Valladolid desde hace unos años con todo lo que rodea al vino, ha sido muy novedosa. Esta explosión del enoturismo y de la apertura de las bodegas de vino al público ha traído consigo que aumente el número de consumidores.

Actualmente, se realizan muchas más actividades relacionadas con el mundo del vino. Hoy día, se pueden encontrar empresas que realicen catas, actividades relacionadas con el enoturismo, etc. Se ha producido un aumento espectacular de tiendas especializadas que organizan actividades relacionadas con el mundo del vino como juegos, cursos de inglés, etc. El diseño y la organización de estos eventos pasan también por encontrar nuevos formatos. 
Carolina apunta que hoy día mucha gente se introduce en el mundo del vino por estar de moda, o también porque están interesados en tener conocimientos nuevos. La sociedad actual en la que vivimos es un público más inquieto y deseoso de conocer todo lo relacionado con la cultura del vino, El consumo de vino no es algo reciente, en la actualidad existe un boom que ha empezado a partir del año 99 como un potencial en bruto, pero con grandes posibilidades para el desarrollo económico y fundamentalmente para el resurgimiento de las zonas rurales que están despobladas e intentar fijar población.

Todos consideran que la promoción que realiza Valladolid sobre su oferta enológica es adecuada pero se podrían más cosas. Carolina añade que en Valladolid se debería centrar más la promoción no sólo en lo relacionado con el vino, también en otros productos turísticos muy importantes: congresos, ferias, rutas culturales, la lengua etc. para conseguir una buena promoción de Valladolid habría que unificar todas estas iniciativas para conseguir un objetivo común: elevar las actividades del vino a un escalón más alto.

\section{CONCLUSIONES}

El enoturismo o turismo enológico es aquel tipo de turismo dedicado a gestionar y potenciar la riqueza vitivinícola, está vinculado a conocer bodegas, la historia y saborear los vinos de la región. Los turistas conocen cada zona vitivinícola a través de la degustación de sus vinos y la visita a bodegas y viñedos. Esta situación conlleva la aparición de un nuevo turismo desconocido hasta hace unos años para algunos, pero cada vez más importante para otros.

A pesar de las dificultades económicas a nivel mundial, este mercado continúa creciendo gracias al aumento de la demanda de una nueva generación de consumidores que tienen mucho interés en vino, la Generación del Milenio (personas de ambos sexos con edades comprendidas entre los 12 y los 32 años). No aceptan las tradiciones establecidas sobre vinos, son aventureros y para ellos el vino no es sólo una bebida, si no algo que va más allá.

El panorama vitivinícola español está siendo testigo de la llegada de grandes inversiones al sector en forma de infraestructuras y tecnología con la construcción de numerosas bodegas. No obstante el vino español padece muchos y peligrosos inconvenientes: cae el consumo, pierde popularidad al ser considerado como una bebida alcohólica, se ve penalizado por el precio que adquiere en los restaurantes, se estancan las exportaciones y reconocen los propios productores que no saben venderse bien. Pero lo que hace que todo lo anterior sea sumamente grave es una tremenda sobreproducción con millones de litros que no se mueven.

El sector vitivinícola español es de gran importancia tanto por el valor económico que genera como por la población que ocupa. La proliferación de bares y tabernas con vinos por copas, la ampliación de las cartas de vinos de algunos restaurantes, el aumento del consumo de vino con denominación de origen, el "boom" de la 
gastronomía y su estrecha relación con el vino, son una muestra de los cambios que han tenido lugar en los últimos años en este sector.

En Castilla y León todas las personas, proyectos, ideas, negocios... tienen cabida y son bien recibidos. El carácter abierto de sus gentes e instituciones convierten a esta región en una tierra de oportunidades. Más allá del concepto de la responsabilidad y de la solidaridad está el del compromiso. Un compromiso que se caracteriza por su carácter duradero en el tiempo. Castilla y León es una Comunidad que persevera en ayudar y colaborar con sus gentes y que se encuentra realmente comprometida con el crecimiento y la mejora de las localidades.

Vino de la Tierra de Castilla y León es una Indicación geográfica utilizada para designar los vinos de mesa elaborados con uvas producidas en la Comunidad Autónoma de Castilla y León. Bajo esta indicación protegida se elaboran vinos tintos, blancos y rosados. Esta figura incluye a los viñedos enmarcados en las denominaciones de origen castellanoleonesas, en menciones de Vino de la Tierra, o a productores con derecho a utilizar la mención geográfica.

La crisis económica ha producido un descenso del consumo de vinos a nivel mundial que afectó a los principales países productores, entre ellos España. Las denominaciones de Origen de Castilla y León con mayor volumen de comercialización no se libraron de esta disminución de las ventas en mercados extranjeros.

Valladolid se ha convertido en un referente entre las ciudades del vino gracias a su provincia al ser la única ciudad que aglutina cinco denominaciones de origen. Ha conservado su memoria del vino presente desde su fundación. Muchos de los monasterios que promueven la cultura vitivinícola se han mantenido hasta hoy en día, la mayoría de las bodegas que existen en la actualidad vertebran su existencia en las posadas y tabernas de la antigüedad.

Valladolid es una ciudad, que con el paso del tiempo se ha convertido en una de las ciudades de referencia del vino a nivel nacional. Cuenta con una situación privilegiada en el panorama enoturístico español, gracias a las cinco denominaciones de origen vitivinícolas con las que cuenta. En cada una de sus zonas vitivinícolas existen multitud de bodegas, desde las históricas a las más vanguardistas, restaurantes, alojamientos que ofrecen estancias especiales, tiendas de vino, museos, artesanía, fiestas y tradiciones, catas diferentes... Una variada oferta que despierta el interés del viajero acercándole al interesante mundo del vino.

La importancia de infraestructuras y comunicaciones con las que cuenta Valladolid, (Ave, aeropuerto y una excelente red de autovías), ofrecen todo tipo de facilidades de conexión de Castilla y León tanto a nivel nacional como internacional.

En España cabe reseñar dos grupos de consumidores bastante diferenciados por su edad y educación. En el primero hay una mayor proporción de jóvenes con un bajo conocimiento sobre el vino. Valoran que el vino proceda de una D. O. y su idoneidad 
con la comida con la que se vaya a acompañar. El otro segmento, está conformado por hombres adultos, con un nivel medio y alto de educación, con alto nivel de conocimiento sobre el vino. Este segmento valora la etiqueta, la idoneidad del vino con la comida a la que acompaña y el haberlo probado anteriormente.

Es evidente la proliferación de eventos enoturísticos en los últimos años en la ciudad de Valladolid, tras lo expuesto en los resultados de las entrevistas la ciudad de Valladolid por su componente histórico-artístico-cultural, estructura urbanística, económica y posibilidades turísticas se totalmente adecuada para acoger un evento enoturístico de gran envergadura y ser una de las ciudades referentes en el panorama español, europeo e internacional.

\subsection{Contraste de Hipótesis.}

Esta investigación partía de unas hipótesis iniciales, las cuales se verán refutadas o verificadas mediante una serie de argumentaciones resultantes del análisis. A modo de recordatorio, las hipótesis planteadas eran las siguientes:

1. Valladolid se concibe como capital idónea de la comunidad de Castilla y León para desarrollar el festival del vino.

Tras el estudio realizado se comprueba que esta hipótesis se cumple debido a varias razones. Valladolid reúne las características adecuadas para que un evento de esta magnitud y temática se pueda llevar a cabo. Es una ciudad moderna, que con el paso del tiempo se ha convertido en una de las de referencias del vino a nivel nacional. Cuenta con una situación privilegiada en el panorama enoturístico español, gracias a las cinco denominaciones de origen vitivinícolas con las que cuenta. Asimismo, cuenta con una red muy moderna de infraestructuras de comunicación, transporte y servicios, y a la cabeza el Tren de Alta Velocidad que une Valladolid con Madrid en tan sólo 55 minutos.

En cada una de sus zonas vitivinícolas existen multitud de bodegas, desde las históricas a las más vanguardistas. Existen rutas por cada una de las Denominaciones de Origen que recorren la provincia, Ribera de Duero, Rueda, Cigales, Toro y Tierras de León. La Ruta Urbana enogastronómica es una oportunidad para conocer el Valladolid histórico combinando lo mejor del Patrimonio cultural de la ciudad con las zonas de vinos y pinchos. Las "Tapas" han dado a la gastronomía española fama internacional y Valladolid ostenta el título indiscutible de Capital de la Tapa.

Todos los años se dan cita en la ciudad los más destacados chefs para competir en el Concurso Nacional de Pinchos y Tapas Ciudad de Valladolid que es una de sus señas de identidad, convirtiéndose de esta manera en un referente gastronómico a nivel nacional.

2. Los nuevos grupos de consumo de vino han creado nuevos estereotipos en la sociedad, que crean otras tendencias destinadas especialmente a este sector. 
Respecto a esta segunda hipótesis, también se cumple por diferentes motivos. Los nuevos estereotipos de consumo han hecho que se desarrollen diferentes tendencias específicas para atender las distintas necesidades de estos grupos cada vez más mayoritarios y que exigen una atención más personalizada.

El crear cultura del vino es un proceso lento, no se mide en años, se mide en generaciones, el que la gente tenga su propio criterio, su propio gusto y opinión, es todavía muy difícil. Dominan la opinión pública una serie de gurús que en cierto modo marcan las últimas tendencias en el mundo del vino.

Los hábitos de consumo españoles han sufrido importantes transformaciones en las últimas décadas, una de las cuales ha afectado sensiblemente a su conducta ante el consumo de vino, que implica cambios relevantes tanto cualitativos como cuantitativos.

En la actualidad las tendencias vitivinícolas que empiezan a producirse y que continuarán en un son muy novedosas. Antes, simplemente se limitaban a producir vino y venderlo, ahora la tendencia que se lleva desde hace unos años es introducirse en las diferentes bodegas y profundizar en el Marketing experiencial del vino. En la actualidad, las bodegas que apuestan por el enoturismo enseñan al mundo todo el proceso del vino y actividades directamente relacionadas con el disfrute del mismo.

\section{REFERENCIAS}

Alet, J. (1996). Marketing Relacional. Barcelona: Gestión 2000.

Alonso, C. (2009). El funcionamiento de las Agencias especializadas de Enoturismo. Ponencia presentada en la $2^{a}$ Edición del Salón Internacional del Turismo del Vino DestinoVino. Abril 13, Logroño.

Altés, C. (1993). Marketing y turismo. Madrid: Editorial Síntesis.

Burgos, D. y León, L. (2001). Comercio electrónico, publicidad y marketing en Internet. Madrid: McGraw - Hill.

Campo, J. (2009). Nuevas formas de Turismo. Madrid: Editorial Síntesis.

Cerrada, A. (2010). Comunicación Turística. Madrid: Esic.

Cervera, A.L. (2006). Comunicación Total. Madrid: Esic.

Del Rey, R (2010). El Enoturismo y sus estrategias. Madrid: RA-MA.

Gómez, A. (2006). Marketing relacional directo e interactivo. Madrid: RA-MA Editorial. 
Llorca, G. (2009). "La Comunicación interna en la comunicación corporativa". Serie Actualidad en Relaciones Públicas, 107-116.

Wagner, R. (2008). Estudios del Consumidor en Enoturismo. Madrid: McGraw - Hill.

\subsection{Documentos electrónicos}

"Actividades enológicas llevadas a cabo por la diputación" (2011). Extraída el 24/III/2012. Diputación Provincial de Valladolid.

http://www.diputaciondevalladolid.es

Guía del turismo del vino en España 2007. Barcelona: Anaya Touring Club.

“Burdeos". Extraída el 08/02/2011 desde http://www.vinos-fr.com/bordeaux.

"El Bus Vino recorre viñedos de Toro, Urueña y Montealegre". (2011). El Norte de Castilla. Valladolid. Extraída el 02/04/2011.

http:/ / www.elnortedecastilla.es/v/20110129/valladolid/vino-recorrera-vinedos toro-20110129.html

"Enoturismo: Saborear y descansar" (2011). Extraída el 19/03/2011. Ayuntamiento de Valladolid. http://www.info.valladolid.es/web/culturayturismo/zonas-de-vinos

“Enología, enoturismo, vitivinícola y turismo enológico". Extraída el 13/04/2011 desde http://www.diccionariodelvino.com.

Muñiz, R. (2011): “Las técnicas de Análisis de Contenido: Una revisión actualizada" Centro de estudios andaluces. Extraída el 08/06/2011 desde http://public.centrodeestudiosandaluces.es/pdfs/S200103.pdf

"Situación de los vinos con denominación de origen de Castilla y León". (2011). Junta de Castilla y León. Agricultura y ganadería. Extraída el 03/03/2011 www.jcyl.es/web/jcyl/AgriculturaGanaderia/es/Plantilla100Detalle/1246464862173 L_/1272896477299/Comunicacion?plantillaObligatoria=PlantillaContenidoNoticiaHo me

\section{Mónica Matellanes Lazo}

Licenciada (2001) en Publicidad y RR.PP. por la Universidad Complutense de Madrid y Doctora (2009) por la Universidad de Valladolid en Comunicación del Enoturismo. Ha trabajado en Comunicación y Marketing dentro del área vitivinícola y actualmente es profesora agregada en Comunicación y Marketing en la Universidad Europea Miguel de Cervantes de Valladolid. Varias veces profesor erasmus y congresista en Universidades extranjeras de Portugal (Lisboa, Ponte de Lima) y en Reino Unido (Aberystwyth). Autor de varios artículos de investigación en el área de Comunicación 
y la Web 2.0, últimas apariciones en Marketing y Ventas, Vivat Academia, Revista Deusto \& Harvard, Razón y Palabra. 